Pecvnia, Monográfico (2009), pp. 263-278

\title{
Las diferencias de cambio
}

\author{
Cristina Gutiérrez López \\ cristina.gutierrez.lopez@unileon.es \\ Universidad de León \\ Fac. de Ciencias Económicas y Empresariales \\ Campus de Vegazana, $\mathrm{s} / \mathrm{n}$ \\ 24071 León (España)
}

\section{INTRODUCCIÓN Y NORMATIVA}

En el Nuevo Plan General Contable (NPGC) el tratamiento de las diferencias de cambio sigue los parámetros de la norma registro y valoración (NRV) "11. Moneda extranjera" o NRV 13 (Plan Pymes). En el caso de la normativa internacional, se recoge en la NIC "21. Efectos de las variaciones en los tipos de cambio de la moneda extranjera".

Pese a que en el Plan 1990 sólo se hacía referencia al euro y la moneda extranjera, la NRV 11 considera la moneda funcional (moneda del entorno económico principal donde opera la empresa), moneda de presentación (en la que se formularán las cuentas anuales, es decir, el euro) y moneda extranjera. 
Además, en el NPGC desaparece la limitación para el reconocimiento de las diferencias positivas de cambio en ciertos elementos, reconocidos hasta ahora como ingresos diferidos al no ser "realizados". Actualmente, la cuestión estriba en considerar los resultados, ya sean positivos o negativos, como ingresos o gastos a patrimonio neto o llevarlos directamente a pérdidas y ganancias.

NRV 11:

Pueden distinguirse dos claros aspectos abordados en la

- Transacciones en moneda extranjera.

- Conversión de cuentas anuales a la moneda de presentación.

\section{TRANSACCIONES EN MONEDA EXTRANJERA}

Una transacción en moneda extranjera es aquélla cuyo importe se denomina o exige su liquidación en una moneda distinta de la funcional, siendo ésta última la moneda del entorno económico principal en el que opera la empresa, presumiendo pues, salvo prueba en contrario, que la moneda funcional de las empresas domiciliadas en España es el euro ${ }^{1}$.

A los efectos de esta norma, los elementos patrimoniales se diferenciarán, según su consideración (Cuadro 1):

- Partidas monetarias: el efectivo y los activos y pasivos que se vayan a recibir o pagar con una cantidad determinada o determinable de unidades monetarias.

Se incluyen, entre otros, los préstamos y partidas a cobrar, los débitos y partidas a pagar y las inversiones en valores representativos de deuda que cumplan los requisitos anteriores.

- Partidas no monetarias: son los activos y pasivos que no se consideren partidas monetarias, es decir, que se vayan a recibir o pagar con una cantidad no determinada ni determinable de unidades monetarias. Se incluyen, entre otros, los inmovilizados materiales, inversiones inmobiliarias, el fondo de comercio y otros

En el caso del Plan Pymes dicha moneda funcional será necesariamente el euro, siendo éste uno de los requisitos para aplicar este modelo contable. 
inmovilizados intangibles, las existencias, las inversiones en el patrimonio de otras empresas que cumplan los requisitos anteriores, los anticipos a cuenta de compras o ventas, así como los pasivos a liquidar mediante la entrega de un activo no monetario.

\section{Cuadro 1}

\begin{tabular}{|l|l|}
\hline Partidas monetarias & Partidas no monetarias \\
\hline Efectivo & Inmovilizado material \\
Préstamos y partidas a cobrar & Inversiones inmobiliarias \\
Débitos y partidas a pagar & Inmovilizado intangible \\
Títulos de renta fija & Existencias \\
& Títulos de renta variable \\
& Anticipos a cuenta de compras \\
& Anticipos a cuenta de ventas \\
& Pasivos a liquidar mediante la entrega de un activo no \\
& monetario \\
\hline
\end{tabular}

\subsection{Valoración inicial}

Respecto a la valoración inicial, se efectuará mediante la conversión a moneda funcional, aplicando el tipo de cambio de contado (el de transacciones de entrega inmediata) en la fecha de la transacción (aquella en que se cumplen los criterios para su reconocimiento), si bien se permite utilizar un tipo de cambio medio del período (máximo mensual) si no ha sufrido variaciones significativas.

\subsection{Valoración posterior}

En cuanto a la valoración posterior, ésta diferirá según se trate de partidas monetarias o no monetarias.

\subsubsection{Partidas monetarias}

Al cierre del ejercicio se valorarán aplicando el tipo de cambio de cierre, entendido como el tipo de cambio medio de contado, existente en esa fecha. Las diferencias de cambio que puedan surgir en ese momento, tanto positivas como negativas, irán a pérdidas y ganancias, a través de "768. Diferencias positivas de cambio" y "668. Diferencias negativas de cambio", respectivamente (Ejemplos 1-2). 
En el caso particular de los activos financieros de carácter monetario (renta fija) clasificados como disponibles para la venta, las diferencias de cambio se estimarán como si dichos activos se valoraran al coste amortizado en la moneda extranjera, de forma que las diferencias de cambio serán las resultantes de las variaciones en dicho coste amortizado como consecuencia de las variaciones en los tipos de cambio, independientemente de su valor razonable. Dichas diferencias de cambio así calculadas se reconocerán en la cuenta de pérdidas y ganancias del ejercicio en el que surjan. Por el contrario, otros cambios en el importe en libros de estos activos financieros se reconocerán directamente en el patrimonio neto (Ejemplo 3).

\section{Ejemplo $\mathbf{n}^{0}$ 1.- Partidas monetarias: créditos y débitos}

En noviembre del ejercicio X1 una empresa compró mercaderías por 500\$, y ha vendido por 900\$. Ambas transacciones deben liquidarse el 31-1-X2.

Se conocen los siguientes tipos de cambio:

\begin{tabular}{c|c|c|c} 
& $11-X 1$ & $31-12-X 1$ & $31-1-X 2$ \\
\hline Tipo de cambio $(€ / \$)$ & 0,67 & 0,76 & 0,72
\end{tabular}

Se pide: Registro contable hasta la liquidación de las transacciones.

Solución.-

Transacciones iniciales. Tipo de cambio de contado (fecha transacción). Puede ser el tipo de cambio medio del período (máximo mensual):

\begin{tabular}{|c|c|c|}
\hline $\begin{array}{l}600 \text { Compras de mercaderías }{ }^{2} \\
500 \$ \times 0,67 € / \$\end{array}$ & $a$ & $\begin{array}{l}4004 \text { Proveedores, moneda } \\
\text { extranjera }\end{array}$ \\
\hline $\begin{array}{l}4304 \text { Clientes, moneda extranjera } \\
900 \$ \times 0,67 € / \$\end{array}$ & $\mathrm{a}$ & 700 Ventas mercaderías \\
\hline
\end{tabular}

Al cierre del ejercicio. Tipo de cambio de cierre:

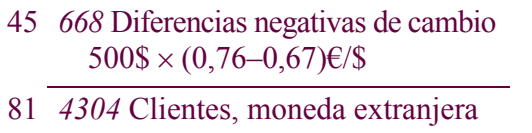

814304 Clientes, moneda extranjera

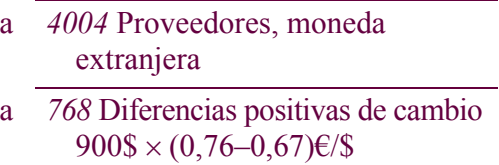

2 En los supuestos prácticos se prescinde de impuestos indirectos recuperables. 
Al liquidarse los débitos y créditos (31-1-X2), las diferencias en la liquidación se producen respecto a las valoraciones de cierre de $\mathrm{X} 1$ :

\begin{tabular}{|l|c|c|c|}
\hline & Valor 31-12-X1 & 31-1-X2 & Diferencia \\
\hline Proveedores & $500 \$ \times 0,76 € / \$=380 €$ & $500 \$ \times 0,72 € / \$=360 €$ & $20 €$ \\
\hline Clientes & $900 \$ \times 0,76 € / \$=684 €$ & $900 \$ \times 0,72 € / \$=648 €$ & $-36 €$ \\
\hline
\end{tabular}

Registro 31-1-X2:

\begin{tabular}{|c|c|c|c|}
\hline \multirow[t]{2}{*}{380} & \multirow{2}{*}{$\begin{array}{l}4004 \text { Proveedores, moneda } \\
\text { extranjera }\end{array}$} & $\mathrm{a}$ & 57 Tesorería \\
\hline & & $\mathrm{a}$ & 768 Diferencias positivas de cambio \\
\hline 648 & 57 Tesorería & $\mathrm{a}$ & 4304 Clientes, moneda extranjera \\
\hline 36 & 668 Diferencias negativas de cambio & & \\
\hline
\end{tabular}

\section{Ejemplo n 2.- Partidas monetarias: tesorería}

Una entidad ingresa en la c/c bancaria 1.200\$ el 12-10-X1. Para ello necesitó 960€. Un año después, retira el dinero de la cuenta.

Se conocen los siguientes tipos de cambio:

\begin{tabular}{c|c|c|c} 
& $12-10-X 1$ & $31-12-X 1$ & $12-10-X 2$ \\
\hline Tipo de cambio (\$/€) & $1.200 \$ / 960 €=1,25$ & 1,28 & 1,2
\end{tabular}

Se pide: Registro contable hasta la liquidación de las transacciones.

\section{Solución.-}

12-10-X1:

$$
9 6 0 \longdiv { 5 7 3 \text { Banco, moneda extranjera } }
$$

A 31-12-X1 el tipo de cambio es de 1,28\$/€.

Así, el valor de la cuenta al cierre es de $1.200 \$ / 1,28 \$ / €=937,5 €$, lo que supone una pérdida de 22,5€:

$$
22,5 0 \longdiv { 6 6 8 \text { Diferencias negativas de cambio } }
$$$$
\text { a } \overline{573 \text { Banco, moneda extranjera }}
$$

El 12-10-X2 el tipo de cambio es de 1,2\$/€.

Por tanto, del valor a 31-12-X1 $(937,5 €)$ a este $1.200 \$ / 1,2 € / \$=1.000 €$ hay diferencias de

\begin{tabular}{|c|c|c|c|}
\hline $1.000,00$ & 572 Banco c/c, $€$ & $\mathrm{a}$ & 573 Banco, moneda extranjera \\
\hline
\end{tabular}
cambio positivas $(62,5 €)$ : 


\section{Ejemplo $n^{0}$ 3.- Partidas monetarias: títulos de renta fija disponibles para la venta}

A comienzos del ejercicio X1, una entidad compra 100 bonos a valor nominal unitario de $200 \$$, siendo el tipo de cambio 0,75€/\$, y los gastos de la operación 250\$. Tipo de interés $5 \%$ pagadero por años vencidos. La amortización será un año después con una prima de reembolso de 10\$/bono. Tipo de cambio al cierre $=0,72 € / \$$. La inversión se califica como disponible para la venta.

Se pide: Registro contable.

\section{Solución.-}

Compra bonos:

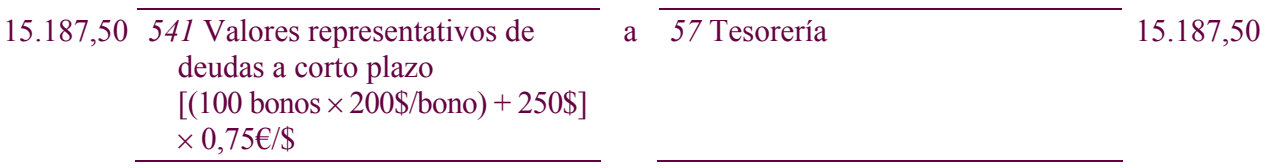

Valoración inicial $=(100$ bonos $\times 200 \$ /$ bono $)+250 \$=20.250 \$$

Determinando el TIE:

$$
20.250=\frac{5 \% 20.000+(100 \times 210)}{(1+i)}=\frac{1.000+21.000}{(1+i)}=\frac{22.000}{(1+i)} \quad i=T I E=8,64 \%
$$

De modo que los intereses devengados en el primer año serán $i \times 20.250=1.750 \$$

31-12-X1 Devengo y cobro de intereses:

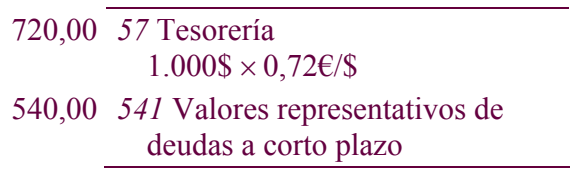
761 Ingresos de valores representativos de deuda $1.750 \$ \times 0,72 € / \$$
$1.260,00$

Amortización de los bonos:

$\begin{aligned} 15.120,00 & \begin{array}{l}57 \text { Tesorería } \\ (100 \text { bonos } \times 210 \$ / €) \times 0,72 € / \$\end{array} \\ 607,50 & \begin{array}{l}668 \text { Diferencias negativas de } \\ \text { cambio }\end{array} \\ & \end{aligned}$

541 Valores representativos de deudas a corto plazo $15.727,50$

\subsubsection{Partidas no monetarias}

2.2.2.1. Valoradas a coste histórico: se valorarán aplicando el tipo de cambio de la fecha de la transacción.

- Cuando el activo se amortice, las dotaciones a la amortización se calcularán sobre el importe en moneda funcional $(€)$ aplicando el tipo de cambio de la fecha en que fue registrado inicialmente. 
Esa valoración no podrá exceder, en cada cierre posterior, el importe recuperable en ese momento, aplicando a este valor, si fuera necesario, el tipo de cambio de cierre (Ejemplos 4-6).

- Cuando, de acuerdo con lo dispuesto en la norma relativa a instrumentos financieros, se deba determinar el patrimonio neto de una empresa participada corregido, en su caso, por las plusvalías tácitas existentes en la fecha de valoración, se aplicará el tipo de cambio de cierre al patrimonio neto y a las plusvalías tácitas existentes a esa fecha ${ }^{4}$ (Ejemplo 7).

2.2.2.2. Valoradas a valor razonable: se valorarán aplicando el tipo de cambio de la fecha de determinación del valor razonable.

- Si los resultados por los cambios en valoración se llevan a patrimonio neto, así se hará también con toda diferencia de cambio incluida en dichos cambios. Es el caso de los activos financieros disponibles para la venta (Ejemplo 8).

- Si los resultados por cambios en valoración se reconocen en la cuenta de pérdidas y ganancias del ejercicio, toda diferencia de cambio incluida también se reconocerá en el resultado del ejercicio. Es el caso de las inversiones en instrumentos de patrimonio clasificadas como activos financieros mantenidos para negociar o en otros activos financieros a valor razonable con cambios en la cuenta de pérdidas y ganancias (Ejemplo 9).

\section{Ejemplo ${ }^{0}$ 4.- Partidas no monetarias a coste histórico: Inmovilizado}

La empresa ha adquirido una máquina 1-1-X1 por 4.000\$, siendo el tipo de cambio vigente en la fecha de compra 0,75€/\$. La máquina se amortizará linealmente en 5 años. A 31-12-X1 el tipo de cambio vigente es $0,72 € / \$$. El valor recuperable son $3.300 \$$.

Se pide: registro contable durante el ejercicio $X 1$.

4 No obstante, si se tratase de empresas extranjeras que se encuentren afectadas por altas tasas de inflación, los citados valores a considerar deberán resultar de estados financieros ajustados, con carácter previo a su conversión. Los ajustes se realizarán de acuerdo con los criterios incluidos sobre "Ajustes por altas tasas de inflación" en las Normas para la Formulación de las Cuentas Anuales Consolidadas, que desarrollan el Código de Comercio. 


\section{Solución.-}

La máquina figurará por $4.000 \$ \times 0,75 € / \$=3.000 €$

Amortización:

\begin{tabular}{cc} 
& $\begin{array}{l}681 \text { Amortización del } \\
\text { inmovilizado material } \\
3.000 / 5\end{array}$ \\
\hline
\end{tabular}

281 Amortización acumulado del inmovilizado material

600,00

31-12-X1:

Valor contable $\$=4.000-4.000 / 5=4.000-800=3.200 \$$

Valor contable $€=3.000-600=2.400 €$

Valor recuperable $€=3.300 \$ \times 0,72 € / \$=2.376 €$

Según NRV 11 la amortización se calculará sobre el importe en moneda funcional $(€)$ aplicando el tipo de cambio en la fecha de registro inicial. El valor así obtenido (2.400€) no puede exceder del importe recuperable calculado a tipo de cambio de cierre (2.376€).

Diferencia de valor $=2.376-2.400=24$ que supone una pérdida para X1:
24,00691 Pérdidas por deterioro del inmovilizado material

291 Deterioro de valor del inmovilizado material

El límite de valoración al final del período es el valor recuperable neto del bien (2.376).

\section{Ejemplo $\mathrm{n}^{0}$ 5.- Partidas no monetarias a coste histórico: existencias (I)}

Una entidad posee a 31-12-X1 una partida de cristal de roca adquirido en Suiza. El importe, en la fecha de adquisición, totalizó 20.000 francos suizos (CHF), siendo el cambio en ese momento 0,68€/CHF.

Al cierre del ejercicio quedan en el almacén todas las unidades compradas, valorándose en el mercado a 20.100CHF, siendo la cotización de 0,66E/CHF.

Se pide: Operaciones de cierre de ejercicio.

\section{Solución.-}

Valor en libros $=20.000 \mathrm{CHF} \times 0,68 € / \mathrm{CHF}=13.600 €$

Valor recuperable a 31-12-X1, según tipo de cambio en la fecha

$$
\rightarrow 20.100 \mathrm{CHF} \times 0,66 € / \mathrm{CHF}=13.266
$$

Pérdida por deterioro $=334$

\begin{tabular}{rl}
$13.600,00$ & 30 Existencias \\
& $\begin{array}{l}\text { 693 Pérdidas por deterioro de } \\
\text { existencias }\end{array}$ \\
\hline
\end{tabular}

\begin{tabular}{llr}
$\mathrm{an}_{\mathrm{a}}$ & $\begin{array}{l}61 \text { Variación de existencias } \\
\begin{array}{l}39 \text { Deterioro de valor de las } \\
\text { existencias }\end{array}\end{array}$ \\
\hline
\end{tabular}
$13.600,00$ 


\section{Ejemplo n ${ }^{0}$ 6.- Partidas no monetarias a coste histórico: existencias (II)}

Una entidad realiza las siguientes operaciones de compraventa de mercaderías, con cobro/pago aplazado:

\begin{tabular}{|c|c|c|c|c|c|c|}
\hline \multicolumn{2}{|c|}{ Operación - Fecha } & \multirow{2}{*}{$\frac{\text { Udes. }}{210}$} & \multirow{2}{*}{$\frac{\text { Precio } \$ / u d .}{5}$} & \multirow{2}{*}{$\frac{\text { Total } \$}{1.050}$} & \multirow{2}{*}{$\frac{\text { Tipo cambio } \boldsymbol{\epsilon} / \mathbf{S}}{0,75}$} & \multirow{2}{*}{$\begin{array}{c}\text { Total } \boldsymbol{\epsilon} \\
787,50\end{array}$} \\
\hline Compra & $2-3$ & & & & & \\
\hline Compra & $5-4$ & 180 & 5,3 & 954 & 0,72 & 686,88 \\
\hline Venta & $15-6$ & 150 & 9 & 1.350 & 0,80 & 1.080 \\
\hline Compra & $5-8$ & 90 & 5,2 & 468 & 0,78 & 365,04 \\
\hline Venta & $6-10$ & 160 & 9,3 & 1.488 & 0,77 & $1.145,76$ \\
\hline
\end{tabular}

El tipo de cambio al cierre es de 0,79€/\$. Suponiendo que la empresa aplique el método FIFO de valoración, y que el valor neto realizable a 31-12 sea de 5,7\$/ud,

Se pide: Registro contable hasta el cierre del ejercicio.

\section{Solución.-}

Total compras $=1.050+954+468=2.472 \$ \rightarrow 787,5+686,88+365,04=1.839,42 €$

Total ventas $=1.350+1.488=2.838 \$ \rightarrow 1.080+1.145,76=2.225,76 €$

El tipo de cambio al cierre es de $0,79 € / \$$

Registro de las operaciones:

\begin{tabular}{|c|c|c|c|}
\hline $1.839,42$ & 600 Compras de mercaderías & a & $\begin{array}{l}4004 \text { Proveedores, moneda } \\
\text { extranjera }\end{array}$ \\
\hline $2.225,76$ & 4304 Clientes, moneda extranjera & $\mathrm{a}$ & 700 Ventas de mercaderías \\
\hline
\end{tabular}

Valoración de las partidas monetarias al cierre:

\begin{tabular}{|l|c|c|c|}
\hline & Valor contable & Valor cierre & Diferencia \\
\hline Proveedores & $1.839,42 €$ & $2.472 \$ \times 0,79 € / \$=1.952,88 €$ & $-113,46$ \\
\hline Clientes & $2.225,76 €$ & $2.838 \$ \times 0,79 € / \$=2.242,02 €$ & 16,26 \\
\hline
\end{tabular}

31-12 Ajuste proveedores:
$113,4 6 \longdiv { 6 6 8 \text { Diferencias negativas de } }$ cambio
a 4004 Proveedores, moneda extranjera

Ajuste clientes:
16,26 4304 Clientes, moneda extranjera
a $\overline{768 \text { Diferencias positivas de cambio }}$

¿Deterioro de valor?

$$
\text { Existencias finales }=210+180-150+90-160=170 \text { udes. }
$$

Valor en libros:

$(80$ udes $\times 5,3 \$ /$ ud $\times 0,72 € / \$)+(90$ udes $\times 5,2 \$ /$ ud $\times 0,78 € / \$)=305,28+365,04=670,32 €$

Valor recuperable $=170 \mathrm{udes} \times 5,7 \$ / \mathrm{ud} \times 0,79 € / \$=765,51 €$

No procede diferencia. 


\section{Ejercicio n $7 .-$ Partidas no monetarias a coste histórico: empresa participada}

Una sociedad adquiere el 25\% de otra. La inversión está formada por 20.000 acciones con un nominal de 15\$/acción, adquiridas al 200\%, siendo en esa fecha el tipo de cambio $1,32 \$ / \epsilon$. En el momento de la compra, el valor teórico es de $25 \$$ /acción. El exceso pagado sobre ese importe corresponde a las plusvalías de un inmueble de la compañia.

Al cierre, el tipo de cambio es 1,35\$/€ y valor teórico de las acciones 26\$. La plusvalía se mantiene intacta.

Se pide: Registro contable.

\section{Solución.-}

Por la adquisición:

\begin{tabular}{cccc}
$454.545,45$ & $\begin{array}{c}240 \text { Participaciones a largo plazo } \\
\text { en partes vinculadas }\end{array}$ & & 57 Tesorería \\
& $(20.000$ acciones $\times 15 € /$ acción & & \\
& $\times 200 \%) / 1,32 \$ / €$ & & \\
\hline
\end{tabular}

¿Deterioro de valor al cierre del ejercicio?

\begin{tabular}{|l|c|}
\hline Precio de adquisición & $454.545,45$ \\
\hline Valor teórico + Plusvalías tácitas & $459.259,26$ \\
20.000 acciones $\times(26+(30-25) \$] / 1,35 \$ / €$ & \\
\hline
\end{tabular}

No hay deterioro.

\section{Ejemplo $\mathrm{n}^{0}$ 8.- Partidas no monetarias a valor razonable: activos disponibles para la venta}

Una entidad adquiere en la Bolsa de NY el 19-11-X1 100 acciones de una sociedad, de nominal $25 \$$, cuando cotizan al 130\%, siendo los gastos de la operación 40€, y el tipo de cambio vigente en el momento de la transacción $0,75 € / \$$. Se considera que las acciones compradas son disponibles para la venta.

A 31-12-X1 la cotización de las acciones es del 135\% y el tipo de cambio 0,78€/\$.

Se pide: Registro contable en X1.

\section{Solución.-}

Los activos disponibles para la venta se valoran tanto inicial como posteriormente a valor razonable. Los cambios en el valor razonable se llevan a patrimonio neto (hasta que el activo cause baja en balance o se deteriore, en que pasarán a pérdidas y ganancias).

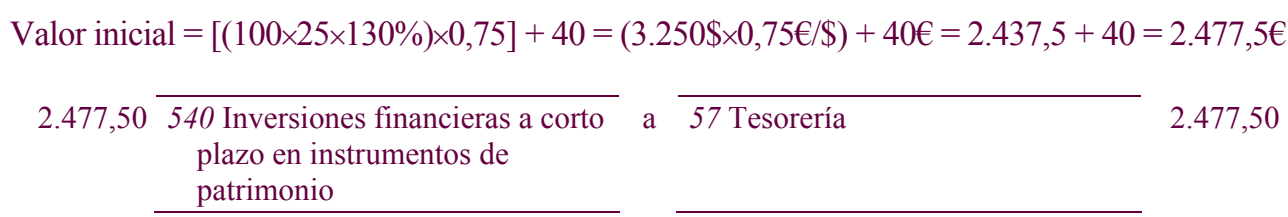




\section{1-12-X1:}

Valoración de acciones: $(100 \times 25 \times 135 \%) \times 0,78=3.375 \times 0,78=2.632,5 €$

Diferencia de valoración $=2.632,5-2.477,5=155$ Ganancia $^{5}$

Esa diferencia puede descomponerse en dos partes: debida a la variación en el valor razonable (coincide con la cotización) y derivada del tipo de cambio.

\section{* Por variación en el valor razonable}

Valor inicial ${ }^{6}=(100 \times 25 \times 130 \%) \times 0,75+40=2.477,5$

Valor final $^{7}=(100 \times 25 \times 135 \%) \times 0,75=2.531,25$

Diferencia $=53,75$ Ganancia

\section{* Por el tipo de cambio}

Valoración final al tipo de cambio antiguo ${ }^{8}=(100 \times 25 \times 135 \%) \times 0,75=2.531,25$

Valoración final al tipo de cambio de cierre $=(100 \times 25 \times 135 \%) \times 0,78=2.632,5$

Diferencia $=101,25$

\begin{tabular}{l}
$155 \begin{array}{l}540 \text { Inversiones financieras a corto } \\
\text { plazo en instrumentos de patrimonio }\end{array}$ \\
\hline
\end{tabular}

a 900 Beneficios en activos disponibles para la venta

\section{Ejemplo $\mathrm{n}^{\circ}$ 9.- Partidas no monetarias a valor razonable: activos mantenidos para} negociar

Se pide: Con los datos del ejemplo anterior, considerar que las acciones se mantienen para negociar.

\section{Solución.-}
155540 Inversiones financieras a corto plazo en instrumentos de patrimonio
7630 Beneficios por valoración de instrumentos financieros por su valor razonable (cartera negociación)

Cartera de negociación: valor inicial a valor razonable (costes de la transacción a pérdidas y ganancias), y valor posterior a valor razonable. Cambios a pérdidas y ganancias.

5 En caso de títulos de renta fija (cuenta 541), se trataría de partidas monetarias, tal que la diferencia por tipo de cambio iría a pérdidas y ganancias (abonando 768 . Diferencias positivas de cambio) y la que se debe al valor razonable, a patrimonio neto (900. Beneficios en activos disponibles para la venta).

6 Cotización inicial y tipo de cambio inicial.

7 Cotización final.

8 Tipo de cambio inicial, cotización final. 


\section{CONVERSIÓN DE LAS CUENTAS ANUALES A LA MONEDA DE}

\section{PRESENTACIÓN}

La moneda de presentación es la moneda en que se formulan las cuentas anuales, es decir, el euro. Excepcionalmente, cuando la moneda o monedas funcionales de una empresa española sean distintas del euro, la conversión de sus cuentas anuales a la moneda de presentación se realizará aplicando los criterios establecidos sobre "Conversión de estados financieros en moneda funcional distinta de la moneda de presentación" en las Normas para la Formulación de las Cuentas Anuales Consolidadas, que desarrollan el Código de Comercio (Cuadro 2).

\section{Cuadro 2}

Criterios establecidos sobre "Conversión de estados financieros en moneda funcional distinta de la moneda de presentación" en las Normas para la Formulación de las Cuentas Anuales Consolidadas, que desarrollan el Código de Comercio, aprobadas por RD 1815/1991, de 20 de diciembre.

Dos métodos de conversión:

\section{Método del tipo de cambio de cierre:}

- Todos los bienes, derechos y obligaciones se convertirán utilizando el tipo de cambio vigente en la fecha de cierre del ejercicio.

- Las partidas de la Cuenta de pérdidas y ganancias se convertirán utilizando los tipos de cambio existentes en las fechas en que se realizaron las correspondientes operaciones. Puede usarse un tipo de cambio medio si se pondera debidamente, según el volumen de las transacciones realizadas.

- La diferencia entre fondos propios, incluido el saldo de la Cuenta de resultados de acuerdo al párrafo anterior, convertidos al tipo de cambio histórico, y la situación patrimonial neta después de conversión de bienes, derechos y obligaciones mencionada antes se llevará a la partida "diferencias de conversión". De acuerdo al NPGC, las diferencias de conversión aparecen en Patrimonio neto.

2. Método monetario-no monetario:

- Partidas no monetarias del Balance: tipo de cambio histórico (el vigente en la fecha en que cada elemento pasó a formar parte del patrimonio de la entidad). Las correcciones valorativas de elementos no monetarios, con el tipo de cambio aplicable a los correspondientes elementos.

- Partidas monetarias del Balance: tipo de cambio vigente en fecha de cierre.

- Partidas de la Cuenta de pérdidas y ganancias: tipo de cambio vigente en la fecha en que se realizaron las correspondientes transacciones. Puede usarse tipo de cambio medio ponderado debidamente, según volumen de transacciones realizadas por cada periodo para evitar la incidencia de la estacionalidad.

- Ingresos y gastos relacionados con partidas no monetarias (dotaciones a amortización, deterioros, resultado por enajenación de elementos no monetarios...) a tipo de cambio histórico aplicado a esas partidas no monetarias.

- Diferencia: a resultados. 
Las diferencias de conversión se registrarán directamente en el patrimonio neto.

Cuando una empresa española sea partícipe en activos o explotaciones en el extranjero controlados conjuntamente según se definen en la norma relativa a negocios conjuntos y la moneda funcional de esos negocios no sea el euro, se seguirán los procedimientos de conversión a moneda de presentación indicados anteriormente. Para los negocios conjuntos que se integren en las cuentas anuales del partícipe, las transacciones en moneda extranjera realizadas por dichos negocios se convertirán a moneda funcional aplicando las reglas contenidas en el apartado primero de esta misma norma. Estos mismos criterios serán aplicables a las sucursales de la empresa en el extranjero.

Ejemplo n ${ }^{0}$ 10.- Conversión de cuentas anuales

La Sociedad Ginebra, constituida el 1-1-X0 y domiciliada en España, opera en un entorno económico cuya moneda funcional es el franco suizo (CHF). Las cuentas anuales de la sociedad a 31-12-X0 son, expresadas en francos suizos:

\begin{tabular}{|c|c|c|c|}
\hline Activo & & Patrimonio neto y Pasivo & \\
\hline Inmovilizado & $80.000,00$ & Capital social ........ & $68.000,00$ \\
\hline Amortización acumulada & $(20.000,00)$ & Prima de emisión & $12.000,00$ \\
\hline Existencias. & $15.000,00$ & Resultado ejercicio & $10.000,00$ \\
\hline Clientes. & $35.000,00$ & Deudas largo plazo. & $25.000,00$ \\
\hline Tesorería & $8.500,00$ & Proveedores ........ & $3.500,00$ \\
\hline 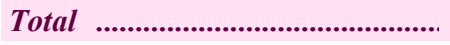 & $118.500,00$ & Total & $118.500,00$ \\
\hline
\end{tabular}

\begin{tabular}{|c|c|}
\hline \multicolumn{2}{|l|}{ Cuenta de Pérdidas y Ganancias } \\
\hline 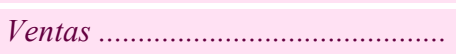 & $105.000,00$ \\
\hline Variación de existencias ..... & $10.000,00$ \\
\hline Otros ingresos ................... & $15.000,00$ \\
\hline Compras ............... & $(43.000,00)$ \\
\hline Amortizaciones ............ & $(20.000,00)$ \\
\hline 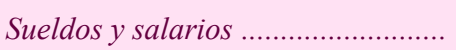 & $(57.000,00)$ \\
\hline Resultado del ejercicio ..................... & $10.000,00$ \\
\hline
\end{tabular}

Se pide: Convertir las cuentas anuales a euros, conociendo los siguientes tipos de cambio $(€ / C H F)$ :

\begin{tabular}{|c|c|}
\hline Creación de la empresa.. & 0,62 \\
\hline Compra de inmovilizado ..... & 0,66 \\
\hline Tipo de cambio medio del ejercicio ..................... & 0,67 \\
\hline Tipo de cambio al cierre ............... & 0,65 \\
\hline Tipo de cambio compra existencias ........ & 0,63 \\
\hline
\end{tabular}




\section{Solución.-}

1) MÉTODO TIPO DE CAMBIO DE CIERRE

- Bienes, derechos y obligaciones al tipo de cambio al cierre:

\begin{tabular}{|l|c|c|c|}
\hline & $\mathbf{C H F}$ & $\mathbf{\epsilon} / \mathbf{C H F}$ & $\boldsymbol{\epsilon}$ \\
\hline Inmovilizado & 80.000 & & 52.000 \\
\hline Amortización acumulada & $(20.000)$ & & $(13.000)$ \\
\hline Existencias & 15.000 & 0,65 & 9.750 \\
\hline Clientes & 35.000 & & 22.750 \\
\hline Tesorería & 8.500 & & 5.525 \\
\hline Total & $\mathbf{1 1 8 . 5 0 0}$ & & $\mathbf{7 7 . 0 2 5}$ \\
\hline
\end{tabular}

- Obligaciones: tipo de cambio al cierre

- Resultado: tipo de cambio medio del ejercicio

- Capital social y prima emisión: tipo de cambio histórico (fecha constitución):

\begin{tabular}{|l|c|c|c|}
\hline Patrimonio neto y Pasivo & $\mathbf{C H F}$ & $\boldsymbol{\epsilon} / \mathbf{C H F}$ & $\boldsymbol{€}$ \\
\hline Capital social & 68.000 & 0,62 & 42.160 \\
\hline Prima emisión & 12.000 & 0,62 & 7.440 \\
\hline Resultado ejercicio & 10.000 & $\mathrm{PyG}$ & 6.700 \\
\hline Deudas a largo plazo & 25.000 & 0,65 & 16.250 \\
\hline Proveedores & 3.500 & 0,65 & 2.275 \\
\hline Resultados positivos de conversión & & & 2.200 \\
\hline & 118.500 & & $74.825+2.200=77.025$ \\
\hline
\end{tabular}

Diferencias de conversión $=77.025-74.825=2.200$

- Cuenta de pérdidas y ganancias: tipo medio del ejercicio:

\begin{tabular}{|l|c|c|c|}
\hline & $\mathbf{C H F}$ & $\boldsymbol{\epsilon} / \mathbf{C H F}$ & $\boldsymbol{\epsilon}$ \\
\hline Ventas & 105.000 & & 70.350 \\
\hline Variación de existencias & 10.000 & & 6.700 \\
\hline Otros ingresos & 15.000 & & 10.050 \\
\hline Compras & $(43.000)$ & 0,67 & $(28.810)$ \\
\hline Amortizaciones & $(20.000)$ & & $(13.400)$ \\
\hline Sueldos y salarios & $(57.000)$ & & $(38.190)$ \\
\hline Resultado del ejercicio & 10.000 & & 6.700 \\
\hline
\end{tabular}

Por tanto, al cierre se registraría:

\begin{tabular}{l}
$2.200 \begin{array}{l}920 \text { Diferencias de conversión } \\
\text { positivas }\end{array}$ \\
\hline
\end{tabular}

a 135 Diferencias positivas de conversión

\section{2) MÉTODO MONETARIO-NO MONETARIO}

\section{- Balance}

Partidas no monetarias: tipo de cambio histórico (Inmovilizado, amortización acumulada, existencias, capital, prima).

Partidas monetarias: tipo de cambio de cierre (Clientes, tesorería, deudas largo plazo, proveedores). 


\begin{tabular}{|l|c|c|c|}
\hline Activo & $\mathbf{C H F}$ & $\boldsymbol{€} / \mathbf{C H F}$ & $\boldsymbol{€}$ \\
\hline Inmovilizado & 80.000 & 0,66 & 52.800 \\
\hline Amortización acumulada & $(20.000)$ & 0,66 & $(13.200)$ \\
\hline Existencias & 15.000 & 0,63 & 9.450 \\
\hline Clientes & 35.000 & 0,65 & 22.750 \\
\hline Tesorería & 8.500 & 0,65 & 5.525 \\
\hline Total & $\mathbf{1 1 8 . 5 0 0}$ & & $\mathbf{7 7 . 3 2 5}$ \\
\hline
\end{tabular}

\begin{tabular}{|l|c|c|c|}
\hline Patrimonio neto y Pasivo & CHF & $\boldsymbol{\epsilon} / \mathbf{C H F}$ & $\boldsymbol{\epsilon}$ \\
\hline Capital social & 68.000 & 0,62 & 42.160 \\
\hline Prima emisión & 12.000 & 0,62 & 7.440 \\
\hline Resultado ejercicio & 10.000 & PyG & 6.900 \\
\hline Deudas a largo plazo & 25.000 & 0,65 & 16.250 \\
\hline Proveedores & 3.500 & 0,65 & 2.275 \\
\hline Resultados positivos de conversión & & & 1.300 \\
\hline & 118.500 & & $75.025+2.300=77.325$ \\
\hline
\end{tabular}

- Cuenta de pérdidas y ganancias

Tipo de cambio medio, salvo a amortizaciones, porque son de partidas no monetarias (tipo histórico de éstas):

\begin{tabular}{|l|c|c|c|}
\hline & $\mathbf{C H F}$ & $\mathbf{\epsilon} / \mathbf{C H F}$ & $\boldsymbol{€}$ \\
\hline Ventas & 105.000 & 0,67 & 70.350 \\
\hline Variación de existencias & 10.000 & 0,67 & 6.700 \\
\hline Otros ingresos & 15.000 & 0,67 & 10.050 \\
\hline Compras & $(43.000)$ & 0,67 & $(28.810)$ \\
\hline Amortizaciones & $(20.000)$ & 0,66 & $(13.200)$ \\
\hline Sueldos y salarios & $(57.000)$ & 0,67 & $(38.190)$ \\
\hline Resultado del ejercicio & 10.000 & & 6.900 \\
\hline
\end{tabular}

En este caso, el ajuste por diferencias de conversión positivas será de $1.300 €$.

\section{PRIMERA APLICACIÓN DEL NPGC}

Dado que ahora las diferencias positivas de moneda extranjera se tienen que llevar a la cuenta de resultados del ejercicio, por lo que para las partidas monetarias procederá un ajuste para dar de baja la cuenta "136. Diferencias positivas en moneda extranjera", a través de su cargo contra el abono de una cuenta de reservas.

En cuanto a la conversión de cuentas anuales a la moneda de presentación, se registran los ajustes correspondientes en las cuentas de activo o pasivo, registrando las diferencias de conversión en patrimonio neto, procederá: 

A Activos/Pasivos
a Activos/Pasivos
B
a 135 Diferencias de conversión
$A-B$

No obstante, en el régimen transitorio se establece que "las diferencias de conversión acumuladas que surjan en la primera aplicación de la NRV 11.2 podrán contabilizarse directa y definitivamente en las reservas voluntarias":
A Activos/Pasivos
a Activos/Pasivos
B
a 113 Reservas voluntarias
$A-B$

\section{BIBLIOGRAFÍA}

AmAdor Fernández, S. y I. Carazo González (2008) Plan General de Contabilidad. Comentarios y casos prácticos. Madrid: Ed. CEF.

Campuzano, M. (2008) "Diferencias de cambio por transacciones en moneda extranjera", Partida doble, núm. 195, pp. 48-55.

INTERNATIONAL ACCOUNTING STANDARDS BOARD (2006) Normas Internacionales de información financiera (NIIF), texto completo de las Normas Internacionales de Información Financiera emitidas a 1 de enero de 2006, traducción al español publicada por CISS-PRAXIS con la autorización de la IASCF.

PÉREZ IGLESIAS, J.M. (2007) "Norma de registro y valoración $11^{\text {a }}$. Moneda extranjera en el Borrador de PGC de 19 de febrero de 2007", CISS Técnica Contable, núm. 699, pp. 39-57.

Rejón LóPEZ, M. (2008) Manual práctico del Nuevo Plan General de Contabilidad 2008 ( $2^{\text {a }}$ edición). Grupo editorial universitario. 